\title{
Genomic analyses identify significant genes and processes in adrenocortical carcinoma cells with overexpressed Ptch1
}

\section{Min Zhang}

Shanghai University

Hongmei Guo

Shanghai University

Hanming Gu (D laygmp@gmail.com )

Shanghai University

James Liu

Shanghai University

\section{Research Article}

Keywords:

Posted Date: February 1st, 2022

DOI: https://doi.org/10.21203/rs.3.rs-1315756/v1

License: (1) This work is licensed under a Creative Commons Attribution 4.0 International License. Read Full License 


\section{Abstract}

Adrenocortical carcinoma is a rare malignancy that mainly comes from family diseases. However, the mechanism and treatment of this cancer are still unclear today. Here, our objective is to determine the significant genes and signaling by analyzing the RNA-seq data from the Ptch1 overexpression cancer cells. The KEGG and GO analyses showed the Calcium signaling pathway and Pathogenic Escherichia coli infection were the key signaling pathways in Ptch1-overexpressed cancer cells. Moreover, we further identified the ten interactive molecules including ALB, STAT3, FOS, NRXN1, SNAP25, SYP, FYN, SPP1, THY1, GRIN2A. Our study may provide insights into the mechanism of Ptch1 regulating adrenocortical carcinoma.

\section{Introduction}

Adrenal tumors are common and benign in the human population, but adrenocortical carcinoma (ACC) is a rare endocrine malignancy ${ }^{1}$. The surveillance database showed the incidence is approximately 0.72 per million cases per year in the USA ${ }^{1}$. The reason for ACC is largely caused by the TP53 mutations, which account for $50 \%-80 \%$ of children with $\mathrm{ACC}^{2}$. Thus, TP53 germline testing is recommended for patients with $\mathrm{ACC}^{3}$. Most ACC patients showed signs of hormone excess, and other patients indicate nonspecific symptoms caused by the tumor growth including the abdominal pain, early satiety or unrelated medical issues ${ }^{4}$. Recently, there are a couple of new modes of treatment such as receptors or enzymes ${ }^{5}$. Unfortunately, these medical trials do not meet the patients' needs due to various side effects.

Ptch1 was detected in various cancers such as lung cancers, colorectal cancers, and breast cancers ${ }^{6}$. There were significant associations between Ptch 1 and Gli1 expression with large tumor size ${ }^{7}$. Moreover, the mediation of Smo activation by Ptch1 is changed in several cancers. Ptch1 inhibits Smo substoichiometrically to control the progression of cancers ${ }^{8}$. Ptch 1 contains a GXXXD motif that is highly conserved in the resistance-nodulation-division family, and changes in GXXXD motif are essential for the activity of oncogene PTC- $3^{9}$. Thus, Ptch1 may be a prognostic marker for high-risk cancer patients.

In our study, we analyzed the impact of Ptch1 on ACC by using the RNA-seq data. We figured out a number of DEGs and significant signaling pathways. We also performed the gene enrichment and created the protein-protein interaction (PPI) network to obtain the interacting signaling map and key molecules. These key genes and pathways in our study may provide novel insights for the treatment of ACC.

\section{Methods}

\section{Data resources}

Gene dataset GSE189424 was downloaded from the GEO database. The data was produced by the Illumina NextSeq 500 (Homo sapiens) (Functional Genomics Platform of Nice-Sophia-Antipolis, 660 route 
des lucioles, Valbonne - Sophia-Antipolis, France). The analyzed dataset includes three controls and three Ptch1 overexpression H295R cell lines.

Data acquisition and processing

The data were organized and conducted by the $\mathrm{R}$ package as previously described ${ }^{10-13}$. We used a classical t-test to identify DEGs with $P<0.01$ and fold change $\geq 1.5$ as being statistically significant.

The Kyoto Encyclopedia of Genes and Genomes (KEGG) and Gene Ontology (GO)

KEGG and GO analyses were conducted by the R package (ClusterProfiler) and Reactome. $P<0.05$ was considered statistically significant.

Protein-protein interaction (PPI) networks

The Molecular Complex Detection (MCODE) was used to create the PPI networks. The significant modules were produced from constructed PPI networks and String networks. The biological processes analyses were performed by using Reactome (https://reactome.org/), and $\mathrm{P}<0.05$ was considered significant.

\section{Results}

\section{Identification of DEGs in ACC cells after overexpression of Ptch1}

To determine the effects of Ptch1 on ACC, we analyzed the RNA-seq data from the ACC cells with the overexpression of Ptch1. A total of 1160 genes were identified with the threshold of $P<0.01$. The top upand down-regulated genes were indicated by the heatmap and volcano plot (Figure 1). The top ten DEGs were listed in Table 1.

\section{Enrichment analysis of DEGs after overexpression of Ptch1 in ACC cells}

To further figure out the potential biological processes in ACC cells with the overexpression of Ptch1, we performed the KEGG and GO analyses (Figure 2). We identified the top ten KEGG signaling pathways, including "Calcium signaling pathway", "Pathogenic Escherichia coli infection”, "Phospholipase D signaling pathway", "Oxytocin signaling pathway", "ECM-receptor interaction”, "Platelet activation”, "Complement and coagulation cascades", "PPAR signaling pathway”, "Arrhythmogenic right ventricular cardiomyopathy", and "Adipocytokine signaling pathway". We identified the top ten BP items of GO enrichment, including "axon development", "axonogenesis", "cell junction assembly", "regulation of nervous system development", "synapse organization", "axon guidance", "neuron projection guidance", "cell-cell adhesion via plasma-membrane adhesion molecules", "regulation of synapse organization", and "regulation of synapse structure or activity". We identified the top ten CC items of GO enrichment, including "synaptic membrane", "collagen-containing extracellular matrix", "membrane raft", "membrane 
microdomain", "neuron to neuron synapse", "postsynaptic density", "asymmetric synapse”, "postsynaptic membrane", "intrinsic component of synaptic membrane", and "intrinsic component of postsynaptic membrane". We also identified the top ten MF items of GO enrichment, including "DNA-binding transcription activator activity, RNA polymerase II-specific", "DNA-binding transcription activator activity", "peptide receptor activity", "cell adhesion mediator activity", "calcium-dependent protein binding", "cell-cell adhesion mediator activity", "scaffold protein binding", "extracellular matrix structural constituent conferring tensile strength", "ionotropic glutamate receptor activity", and "platelet-derived growth factor binding".

\section{PPI network analysis}

To determine the relationship among the DEGs, we create the PPI network by using 867 nodes and 2328 edges (Combined score $>0.2$ as a cutoff, Cytoscope software). Table 2 showed the top ten genes with the highest scores. The top two significant clusters were indicated in Figure 3. We further analyzed the PPI and DEGs with Reactome map (Figure 4) and identified the top ten functional processes including "Response of EIF2AK1 (HRI) to heme deficiency", "Negative regulation of activity of TFAP2 (AP-2) family transcription factors", "Acyl chain remodelling of PG", "Ligand-receptor interactions", "Dissolution of Fibrin Clot", "Defective CHST3 causes SEDCJD", "Collagen degradation", "RUNX3 regulates RUNX1-mediated transcription", "CHL1 interactions", and "Acyl chain remodelling of PS" (Supplemental Table S1).

\section{Discussion}

Ptch1 is the most important Hh signaling regulator in cancers such as colorectal cancer, but the potential relationships between Ptch1 and patients' outcomes are still not clear ${ }^{14}$. Therefore, we herein use the data from the renal cancer cells with the overexpression of Ptch1 to assess the functions of Ptch1, thereby exploring the possible anti-cancer drugs.

By analyzing the KEGG and GO enrichment data, we found the "Calcium signaling pathway" and "Pathogenic Escherichia coli infection" were the key signaling pathways in Ptch1-overexpressed cancer cells. Yingying Hong et al found that Ptch1 siRNA decreases the SPARC levels, which affects the calcium metabolism ${ }^{15}$. Wu-Bo Li et al found that PTCH1 protein is a critical target for regulating the influenza virus infection. Moreover, $\mathrm{PTCH} 1$ was discovered to have association with decreased morbidity during the influenza infection ${ }^{16}$.

Besides the biological signaling, we also identified ten interacting molecules that were affected by the overexpression of Ptch1 in renal cancer cells. Sakae Konishi et al found that C-reactive protein/ALB ratio is a predictive marker for prognosis in cancer patients ${ }^{17}$. Keita Tamura et al also found the utility of the albumin can be considered as an objective prognostication tool to validate the metastatic cell carcinoma patients receiving second-line axitinib ${ }^{18}$. The activation of STAT3 can mediate multiple gene functions such as cell proliferation, differentiation, and apoptosis. Moreover, the inhibition of STAT3 was considered as an important therapy for cancer ${ }^{19}$. Circadian clocks regulate several downstream gene expressions through the transcriptional level to further mediate the cell functions such as proliferation, 
differentiation, apoptosis, cell death, and metabolism ${ }^{20-31}$. Zhenghui Tang et al found that STAT3 can inhibit the CRY2 expression through the CLOCK/BMAL1/P300 signaling ${ }^{32}$. Neil J Manimala et al found that FOS is a cellular proto-oncogene that increases the genes involved in proliferation and cancer formation $^{33}$. Takuma Yotsumoto et al found that NRXN1 is a novel drug target for cancers such as lung cancer $^{34}$. Qiongzhen Huang et al found that SNAP25 can inhibit the glioma progression through mediating synapse plasticity ${ }^{35}$. Diana Rodica Tudoraşcu et al found SYP is considered as a prognostic factor for colorectal cancer ${ }^{36}$. Jörg Ellinger found that synaptophysin expression is associated with shorter cancer-specific survival of renal cancer patients ${ }^{37}$. Daniel Elias et al discovered that FYN is a critical molecule in cancer pathogenesis ${ }^{38}$. GPCR/RGS and their downstream signaling play vital roles in human physiological and pathological conditions, including aging, inflammation, metabolism homeostasis, and nervous system regulation ${ }^{39-50}$. Interestingly, Rithwick Rajagopal et al found that Fyn is activated by GPCR stimulation and is responsible for further activating the Trk receptors on intracellular membranes ${ }^{51}$. Maj Rabjerg et al found SPP1 was identified as a promising novel prognostic marker for cancers ${ }^{52}$. THY1 contains critical roles in cancer, which regulates cancer cell proliferation, metastasis, and angiogenesis ${ }^{53}$. X J Zhou et al found that the expression of GRIN2A showed different clinical significance between benign and malignant cancers ${ }^{54}$.

In summary, our study found a strong relationship between Ptch1 and adrenocortical cancer. The Calcium signaling pathway and Pathogenic infection are the major affected processes in the Ptch1 regulated adrenocortical cancer. Based on these findings, our study provides valuable insights for the diagnosis and treatment of adrenocortical cancer.

\section{Declarations}

\section{Author Contributions}

Min Zhang, Hongmei Guo: Methodology and Writing. Hanming Gu, James Liu: Conceptualization, WritingReviewing and Editing.

\section{Funding}

This work was not supported by any funding.

\section{Declarations of interest}

There is no conflict of interest to declare.

\section{References}

1. Else T, Kim AC, Sabolch A, Raymond VM, Kandathil A, Caoili EM, Jolly S, Miller BS, Giordano TJ, Hammer GD: Adrenocortical carcinoma. Endocr Rev 2014, 35:282-326. 
2. Wasserman JD, Novokmet A, Eichler-Jonsson C, Ribeiro RC, Rodriguez-Galindo C, Zambetti GP, Malkin D: Prevalence and functional consequence of TP53 mutations in pediatric adrenocortical carcinoma: a children's oncology group study. J Clin Oncol 2015, 33:602-9.

3. Sorrell AD, Espenschied CR, Culver JO, Weitzel JN: Tumor protein p53 (TP53) testing and Li-Fraumeni syndrome: current status of clinical applications and future directions. Mol Diagn Ther 2013, 17:3147.

4. Else T, Williams AR, Sabolch A, Jolly S, Miller BS, Hammer GD: Adjuvant therapies and patient and tumor characteristics associated with survival of adult patients with adrenocortical carcinoma. $\mathrm{J}$ Clin Endocrinol Metab 2014, 99:455-61.

5. Hauser AS, Attwood MM, Rask-Andersen M, Schioth HB, Gloriam DE: Trends in GPCR drug discovery: new agents, targets and indications. Nat Rev Drug Discov 2017, 16:829-42.

6. Wang CY, Chang YC, Kuo YL, Lee KT, Chen PS, Cheung CHA, Chang CP, Phan NN, Shen MR, Hsu HP: Mutation of the PTCH1 gene predicts recurrence of breast cancer. Sci Rep 2019, 9:16359.

7. Lo WW, Pinnaduwage D, Gokgoz N, Wunder JS, Andrulis IL: Aberrant hedgehog signaling and clinical outcome in osteosarcoma. Sarcoma 2014, 2014:261804.

8. Onodera S, Nakamura Y, Azuma T: Gorlin Syndrome: Recent Advances in Genetic Testing and Molecular and Cellular Biological Research. Int J Mol Sci 2020, 21.

9. Taipale J, Cooper MK, Maiti T, Beachy PA: Patched acts catalytically to suppress the activity of Smoothened. Nature 2002, 418:892-7.

10. Yu G, Wang LG, Han Y, He QY: clusterProfiler: an R package for comparing biological themes among gene clusters. OMICS 2012, 16:284-7.

11. Hanming G: nuotrophils arthritis. Research Square 2021.

12. Jing L, Letian W, Hanming G: Identification of driver genes and biological signaling for alcoholic myopathy. Research Square 2021.

13. Yuan G: Identification of biomarkers and pathways of mitochondria in sepsis patients. bioRxiv 2021:2021.03.29.437586.

14. Skoda AM, Simovic D, Karin V, Kardum V, Vranic S, Serman L: The role of the Hedgehog signaling pathway in cancer: A comprehensive review. Bosn J Basic Med Sci 2018, 18:8-20.

15. Hong Y, Zhang J, Zhang H, Li X, Qu J, Zhai J, Zhang L, Chen F, Li T: Heterozygous PTCH1 Mutations Impact the Bone Metabolism in Patients With Nevoid Basal Cell Carcinoma Syndrome Likely by Regulating SPARC Expression. J Bone Miner Res 2016, 31:1413-28.

16. Li WB, Zhu J, Hart B, Sui B, Weng K, Chang S, Geiger R, Torremorell M, Mileham A, Gladney C, Mellancamp MA, Li L, Yunus A, Goldblatt M, Kinch MS: Identification of PTCH1 requirement for influenza virus using random homozygous gene perturbation. Am J Transl Res 2009, 1:259-66.

17. Konishi S, Hatakeyama S, Tanaka T, Ikehata Y, Tanaka T, Hamano I, Fujita N, Yoneyama T, Yamamoto H, Yoneyama T, Hashimoto Y, Yoshikawa K, Kawaguchi T, Masumori N, Kitamura H, Ohyama C: C- 
reactive protein/albumin ratio is a predictive factor for prognosis in patients with metastatic renal cell carcinoma. Int J Urol 2019, 26:992-8.

18. Tamura K, Osawa T, Takeuchi A, Minami K, Nakai Y, Ueda K, Ozawa M, Uemura M, Sugimoto M, Ohba K, Suzuki T, Anai S, Shindo T, Kusakabe N, Komiyama M, Tanaka K, Yokomizo A, Kohei N, Shinohara $\mathrm{N}$, Miyake $\mathrm{H}$, Japanese Urological Oncology G: External validation of the albumin, C-reactive protein and lactate dehydrogenase model in patients with metastatic renal cell carcinoma receiving secondline axitinib therapy in a Japanese multi-center cohort. Jpn J Clin Oncol 2021, 51:810-8.

19. Mohassab AM, Hassan HA, Abdelhamid D, Abdel-Aziz M: STAT3 transcription factor as target for anti-cancer therapy. Pharmacol Rep 2020, 72:1101-24.

20. Reppert SM, Weaver DR: Coordination of circadian timing in mammals. Nature 2002, 418:935-41.

21. Yuan G, Hua B, Yang Y, Xu L, Cai T, Sun N, Yan Z, Lu C, Qian R: The Circadian Gene Clock Regulates Bone Formation Via PDIA3. J Bone Miner Res 2017, 32:861-71.

22. Xu L, Cheng Q, Hua B, Cai T, Lin J, Yuan G, Yan Z, Li X, Sun N, Lu C, Qian R: Circadian gene Clock regulates mitochondrial morphology and functions by posttranscriptional way. bioRxiv 2018:365452.

23. Zhu Z, Hua B, Shang Z, Yuan G, Xu L, Li E, Li X, Sun N, Yan Z, Qian R, Lu C: Altered Clock and Lipid Metabolism-Related Genes in Atherosclerotic Mice Kept with Abnormal Lighting Condition. Biomed Res Int 2016, 2016:5438589.

24. Yuan G, Hua B, Cai T, Xu L, Li E, Huang Y, Sun N, Yan Z, Lu C, Qian R: Clock mediates liver senescence by controlling ER stress. Aging 2017, 9:2647-65.

25. Fan XF, Wang XR, Yuan GS, Wu DH, Hu LG, Xue F, Gong YS: [Effect of safflower injection on endoplasmic reticulum stress-induced apoptosts in rats with hypoxic pulmonary hypertension]. Zhongguo Ying Yong Sheng Li Xue Za Zhi 2012, 28:561-7.

26. Cai T, Hua B, Luo D, Xu L, Cheng Q, Yuan G, Yan Z, Sun N, Hua L, Lu C: The circadian protein CLOCK regulates cell metabolism via the mitochondrial carrier SLC25A10. Biochim Biophys Acta Mol Cell Res 2019, 1866:1310-21.

27. Zhu Z, Xu L, Cai T, Yuan G, Sun N, Lu C, Qian R: Clock represses preadipocytes adipogenesis via GILZ. J Cell Physiol 2018, 233:6028-40.

28. Mao SZ, Fan XF, Xue F, Chen R, Chen XY, Yuan GS, Hu LG, Liu SF, Gong YS: Intermedin modulates hypoxic pulmonary vascular remodeling by inhibiting pulmonary artery smooth muscle cell proliferation. Pulm Pharmacol Ther 2014, 27:1-9.

29. Yuan G, Xu L, Cai T, Hua B, Sun N, Yan Z, Lu C, Qian R: Clock mutant promotes osteoarthritis by inhibiting the acetylation of NFkappaB. Osteoarthritis Cartilage 2019, 27:922-31.

30. Fu L, Pelicano H, Liu J, Huang P, Lee C: The circadian gene Period2 plays an important role in tumor suppression and DNA damage response in vivo. Cell 2002, 111:41-50.

31. Zhu Z, Hua B, Xu L, Yuan G, Li E, Li X, Sun N, Yan Z, Lu C, Qian R: CLOCK promotes 3T3-L1 cell proliferation via Wnt signaling. IUBMB Life 2016, 68:557-68. 
32. Tang Z, Xu T, Li Y, Fei W, Yang G, Hong Y: Inhibition of CRY2 by STAT3/miRNA-7-5p Promotes Osteoblast Differentiation through Upregulation of CLOCK/BMAL1/P300 Expression. Mol Ther Nucleic Acids 2020, 19:865-76.

33. Manimala NJ, Frost CD, Lane ML, Higuera M, Beg R, Vesely DL: Cardiac hormones target nuclear oncogenes c-Fos and c-Jun in carcinoma cells. Eur J Clin Invest 2013, 43:1156-62.

34. Yotsumoto T, Maemura K, Watanabe K, Amano Y, Matsumoto Y, Zokumasu K, Ando T, Kawakami M, Kage H, Nakajima J, Yatomi Y, Nagase T, Takai D: NRXN1 as a novel potential target of antibody-drug conjugates for small cell lung cancer. Oncotarget 2020, 11:3590-600.

35. Huang Q, Lian C, Dong Y, Zeng H, Liu B, Xu N, He Z, Guo H: SNAP25 Inhibits Glioma Progression by Regulating Synapse Plasticity via GLS-Mediated Glutaminolysis. Front Oncol 2021, 11:698835.

36. Tudorascu DR, Pirici D, Tartea EA, Mustafa ER, Florescu C, Vere CC, Balea AM, Puiu I, Tartea GC, Albu VC: Synaptophysin expression as prognostic factor for survival in colorectal carcinomas. Rom J Morphol Embryol 2017, 58:1409-15.

37. Ellinger J, Bastian PJ, Hauser S, Biermann K, Muller SC: Primitive neuroectodermal tumor: rare, highly aggressive differential diagnosis in urologic malignancies. Urology 2006, 68:257-62.

38. Elias D, Ditzel HJ: Fyn is an important molecule in cancer pathogenesis and drug resistance. Pharmacol Res 2015, 100:250-4.

39. Xie K, Martemyanov KA: Control of striatal signaling by g protein regulators. Front Neuroanat 2011, 5:49.

40. Yuan G, Yang S, Ng A, Fu C, Oursler MJ, Xing L, Yang S: RGS12 Is a Novel Critical NF-kappaB Activator in Inflammatory Arthritis. iScience 2020, 23:101172.

41. Yuan G, Yang S, Gautam M, Luo W, Yang S: Macrophage regulator of G-protein signaling 12 contributes to inflammatory pain hypersensitivity. Ann Transl Med 2021, 9:448.

42. Rosenbaum DM, Rasmussen SG, Kobilka BK: The structure and function of G-protein-coupled receptors. Nature 2009, 459:356-63.

43. Yuan G, Yang S, Yang S, Ng A, Oursler MJ: RGS12 is a critical proinflammatory factor in the pathogenesis of inflammatory arthritis via acting in Cox2-RGS12-NF kappa B pathway activation loop. J Bone Miner Res: WILEY 111 RIVER ST, HOBOKEN 07030-5774, NJ USA, 2019. pp. $147-$.

44. Yuan G, Yang S, Yang S: Macrophage RGS12 contributes to osteoarthritis pathogenesis through enhancing the ubiquitination. Genes \& Diseases 2021.

45. Fu C, Yuan G, Yang ST, Zhang D, Yang S: RGS12 Represses Oral Cancer via the Phosphorylation and SUMOylation of PTEN. J Dent Res 2020:22034520972095.

46. Stewart A, Fisher RA: Introduction: G Protein-coupled Receptors and RGS Proteins. Prog Mol Biol Transl Sci 2015, 133:1-11.

47. Yuan G, Huang Y, Yang ST, Ng A, Yang S: RGS12 inhibits the progression and metastasis of multiple myeloma by driving $\mathrm{M} 1$ macrophage polarization and activation in the bone marrow microenvironment. Cancer Commun (Lond) 2022, 42:60-4. 
48. Yuan G, Fu C, Yang ST, Yuh DY, Hajishengallis G, Yang S: RGS12 Drives Macrophage Activation and Osteoclastogenesis in Periodontitis. J Dent Res 2021:220345211045303.

49. Tamirisa P, Blumer KJ, Muslin AJ: RGS4 inhibits G-protein signaling in cardiomyocytes. Circulation 1999, 99:441-7.

50. Yuan G, Yang S, Liu M, Yang S: RGS12 is required for the maintenance of mitochondrial function during skeletal development. Cell Discov 2020, 6:59.

51. Rajagopal R, Chao MV: A role for Fyn in Trk receptor transactivation by G-protein-coupled receptor signaling. Mol Cell Neurosci 2006, 33:36-46.

52. Rabjerg M, Bjerregaard H, Halekoh U, Jensen BL, Walter S, Marcussen N: Molecular characterization of clear cell renal cell carcinoma identifies CSNK2A1, SPP1 and DEFB1 as promising novel prognostic markers. APMIS 2016, 124:372-83.

53. Kumar A, Bhanja A, Bhattacharyya J, Jaganathan BG: Multiple roles of CD90 in cancer. Tumour Biol 2016, 37:11611-22.

54. Zhou XJ, Lin BR, Wang Y: Expression of GRIN2A in benign and malignant nasopharyngeal diseases and its clinical significance. Genet Mol Res 2015, 14:17289-95.

\section{Tables 1-2}

Tables 1-2 are available in the Supplementary Files section.

\section{Figures}
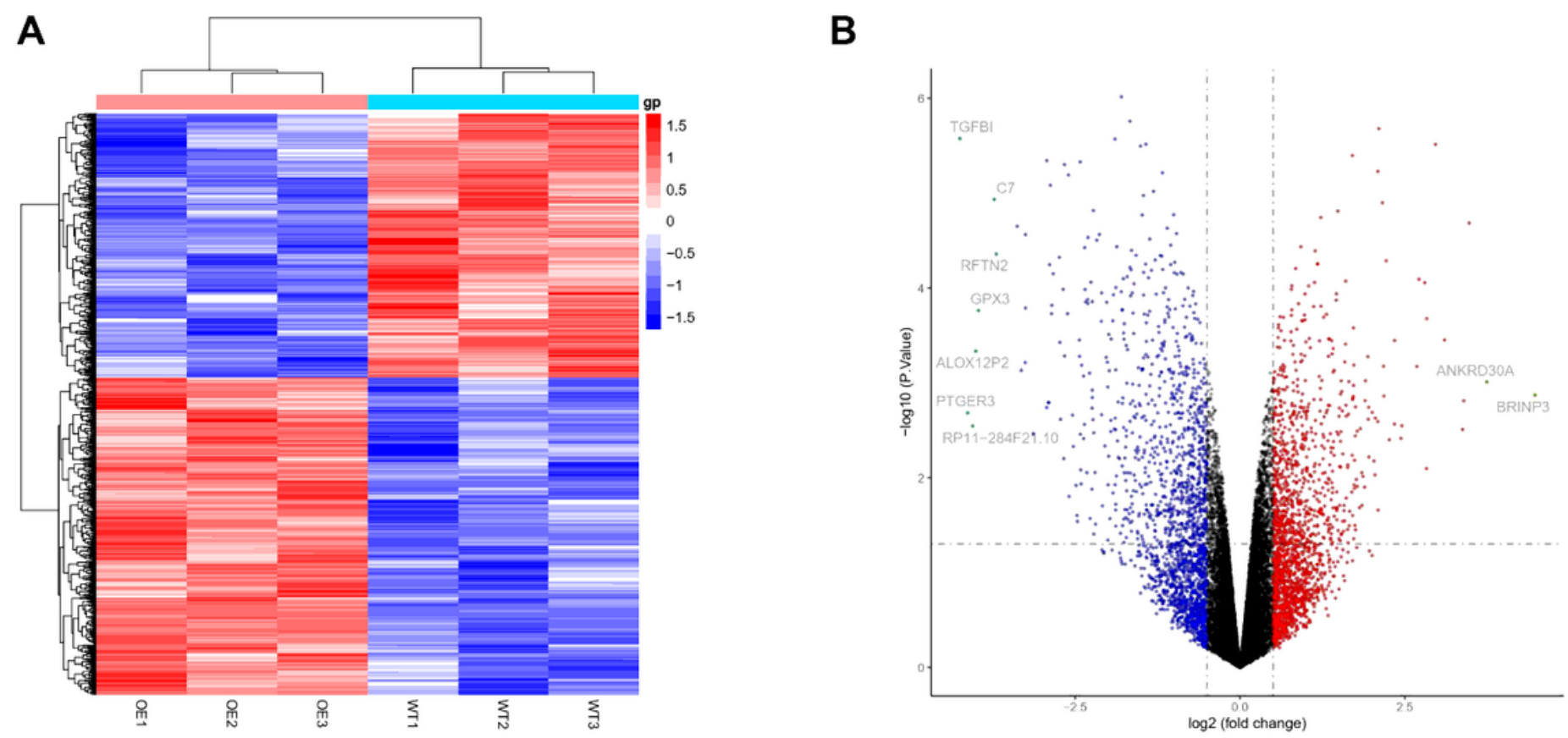

Figure 1 
Heatmap and volcano plot in ACC cells after overexpression of Ptch1

(A) Heatmap of significant DEGs. Significant DEGs $(P<0.01)$ were used to construct the heatmap. WT, wildtype; OE, Ptch1 overexpression.

(B) Volcano plot for DEGs in ACC cells after overexpression of Ptch1. The most significantly changed genes are highlighted by grey dots.

Figure 2

KEGG and GO analyses of DEGs in ACC cells after overexpression of Ptch1

(A) KEGG analysis (B) BP, Biological processes, (C) CC, Cellular components, (D) MF, Molecular functions.

\section{Figure 3}

The PPI network analyses of DEGs in ACC cells after overexpression of Ptch1

The cluster (A) and cluster (B) were constructed by MCODE.

Figure 4

Reactome map representation of the significant biological processes in ACC cells after overexpression of Ptch1 (yellow)

\section{Supplementary Files}

This is a list of supplementary files associated with this preprint. Click to download.

- Onlinefloatimage5.png

- Onlinefloatimage6.png

- SupplementalTableS1.xlsx 